Radio galaxies in galaxy groups : kinematics, scaling relations, and AGN feedback

\title{
Pasini, T.
}

2021-08

Pasini , T , Finoguenov , A , Brueggen , M , Gaspari , M , de Gasperin , F \& Gozaliasl , G 2021 , ' Radio galaxies in galaxy groups : kinematics, scaling relations, and AGN feedback ',

Monthly Notices of the Royal Astronomical Society , vol. 505 , no. 2 , pp. 2628-2637 . https://doi.org/10.1093/mnras/

http://hdl.handle.net/10138/336504

https://doi.org/10.1093/mnras/stab1451

acceptedVersion

Downloaded from Helda, University of Helsinki institutional repository.

This is an electronic reprint of the original article.

This reprint may differ from the original in pagination and typographic detail.

Please cite the original version. 


\title{
Radio galaxies in galaxy groups: kinematics, scaling relations and AGN feedback
}

\author{
T. Pasini ${ }^{1 \star}$, A. Finoguenov ${ }^{2}$, M. Brüggen ${ }^{1}$, M. Gaspari ${ }^{3,4}$, F. de Gasperin ${ }^{1,5}$ and G. Gozaliasl ${ }^{6,2}$ \\ ${ }^{1}$ Hamburger Sternwarte, Universität Hamburg, Gojenbergsweg 112, 21029 Hamburg, Germany \\ ${ }^{2}$ Department of Physics, University of Helsinki, P.O. Box 64, FI-00014 Helsinki, Finland \\ ${ }^{3}$ INAF - Osservatorio di Astrofisica e Scienza dello Spazio, via P. Gobetti 93/3, I-40129 Bologna, Italy \\ ${ }^{4}$ Department of Astrophysical Sciences, Princeton University, 4 Ivy Lane, Princeton, NJ 08544-1001, USA \\ ${ }^{5}$ INAF - Istituto di Radioastronomia, via P. Gobetti 101, 40129, Bologna, Italy \\ ${ }^{6}$ Research Program in Systems Oncology (Oncosys), Faculty of Medicine, University of Helsinki, P.O.Box 63, FI-00014, Helsinki, Finland
}

Accepted 2021 May 17. Received 2021 May 17; in original form 2021 January 20

\begin{abstract}
We investigate the kinematic properties of a large $(\mathrm{N}=998)$ sample of COSMOS spectroscopic galaxy members distributed among 79 groups. We identify the Brightest Group Galaxies (BGGs) and cross-match our data with the VLA-COSMOS Deep survey at $1.4 \mathrm{GHz}$, classifying our parent sample into radio/non-radio BGGs and radio/nonradio satellites. The radio luminosity distribution spans from $L_{R} \sim 2 \times 10^{21} \mathrm{~W} \mathrm{~Hz}^{-1}$ to $L_{R} \sim 3 \times 10^{25} \mathrm{~W} \mathrm{~Hz}^{-1}$. A phase-space analysis, performed by comparing the velocity ratio (line-of-sight velocity divided by the group velocity dispersion) with the galaxy-group centre offset, reveals that BGGs (radio and non-radio) are mostly ( 80\%) ancient infallers. Furthermore, the strongest $\left(L_{R}>10^{23} \mathrm{~W} \mathrm{~Hz}^{-1}\right)$ radio galaxies are always found within $0.2 R_{\mathrm{vir}}$ from the group centre. Comparing our samples with HORIZON-AGN, we find that the velocities and offsets of simulated galaxies are more similar to radio BGGs than to non-radio BGGs, albeit statistical tests still highlight significant differences between simulated and real objects. We find that radio BGGs are more likely to be hosted in high-mass groups. Finally, we observe correlations between the powers of BGG radio galaxies and the X-ray temperatures, $T_{\mathrm{x}}$, and X-ray luminosities, $L_{\mathrm{x}}$, of the host groups. This supports the existence of a link between the intragroup medium and the central radio source. The occurrence of powerful radio galaxies at group centres can be explained by Chaotic Cold Accretion, as the AGN can feed from both the galactic and intragroup condensation, leading to the observed positive $L_{\mathrm{R}}-T_{\mathrm{x}}$ correlation.
\end{abstract}

Key words: galaxies: clusters: general - galaxies: clusters: intracluster medium - galaxies: groups: general - X-rays: galaxies: clusters - radio continuum: galaxies

\section{INTRODUCTION}

The hot plasma inside of galaxy clusters and groups is governed by processes that can be observed at multiple wavelengths: from thermal cooling of the hot $\left(\sim 10^{7} \mathrm{~K}\right)$ intracluster medium (ICM) (e.g., Fabian et al., 1994; Peterson \& Fabian, 2006), to line emission produced by warm gas (e.g., Hamer et al., 2016; Pulido et al., 2018), to feedback from central Active Galactic Nuclei (AGN). The latter can heat their surroundings and can prevent the catastrophic cooling of the cool core (see reviews by, e.g., McNamara \& Nulsen, 2007; Gitti et al., 2012), establishing what is known as $A G N$ feedback cycle.

Brightest Cluster Galaxies (BCGs) and Brightest Group Galaxies (BGGs) are the most optically luminous and massive galaxies in a cluster and group, respectively. Usually they lie at the centres of their host structures. Owing to their spe-

\footnotetext{
* E-mail: thomas.pasini@hs.uni-hamburg.de
}

cial location, the evolution and assembly history of massive galaxies and of their hosts has been studied widely (Bernstein \& Bhavsar, 2001; Bernardi, 2007; Liu et al., 2009; Stott et al., 2010). Notably, BGGs look dissimilar from other massive galaxies, showing different surface brightness profiles and obeying different scaling relations, which suggests that their formation process may be different, too (see e.g., Von Der Linden et al., 2007; Liu et al., 2008; Stott et al., 2008; Shen et al., 2014).

In galaxy clusters, the evolution of BCGs is tightly linked to that of the host cluster (e.g. Lin \& Mohr, 2007). Several observational studies (e.g., Giodini et al., 2010; Giacintucci et al., 2011; Ineson et al., 2013, 2015; Kolokythas et al., 2018; Pasini et al., 2020) and numerical simulations (Gaspari et al. 2020 for a review) have demonstrated the importance of AGN feedback in galaxy groups, that are known to be the hosts of more than half of all galaxies (Eke et al., 2006). However, there is no consensus on where to draw the boundary between galaxy clusters and galaxy groups. It is sometimes 
assumed that the boundary lies close to the virial temperature of $1 \mathrm{keV}$ since this is the temperature where the slope of the relation between X-ray luminosity and virial temperature changes. However, this change of slope could be caused by observational systematics (see e.g., Voit et al., 2018).

The trigger mechanism for radio-loud AGN activity is still unclear (e.g., Shakura \& Sunyaev, 1973; Merloni \& Heinz, 2007; Best \& Heckman, 2012), but gas that cools out of the hot X-ray halo seems to play a key role (e.g., Best et al. 2005). In the Chaotic Cold Accretion (CCA) scenario (Gaspari et al., 2013; Gaspari, 2016) the AGN is frequently switched on and off through self-regulated feeding and feedback cycles. CCA can occur in every galaxy with a hot halo, regardless of the position. However, central galaxies - BGGs or not lie in dense regions, where the condensation is significantly stronger (Gaspari et al., 2019). Non-linear thermal instabilities produced by the cooling plasma lead to precipitation, which is able to feed the SMBH through inelastic collisions between the condensed cold clouds and filaments (e.g., Gaspari \& Sądowski 2017; McDonald et al. 2018; Tremblay et al. 2018; Temi et al. 2018; Juráňová et al. 2019; Rose et al. 2019; Schellenberger et al. 2020).

Pasini et al. (2020) presented a study of the relation between the ICM X-ray luminosity of a sample of 247 X-ray selected galaxy groups in COSMOS (Gozaliasl et al., 2019) and the radio luminosity produced by the corresponding central radio galaxy, defined as the radio source at $1.4 \mathrm{GHz}$ found closest to the X-ray emission peak. Cross-matching this sample with optical catalogs, they found that only in 30 per cent of the groups central radio galaxies were hosted in BGGs. This is consistent with Gozaliasl et al. (2019), who showed that 70 per cent of COSMOS BGGs are found more than $0.1 R_{200}$ away from the X-ray peak. This suggests that BGGs do not always lie at the bottom of the potential well. This does not seem to be the case for galaxy clusters, where $\sim 85$ per cent of central radio galaxies were found in BCGs (Pasini et al., 2020). Nevertheless, there are some cases in which an apparently brightest galaxy near a cluster centre has a significantly large velocity offset with respect to the mean redshift of cluster members (> $300 \mathrm{~km} \mathrm{~s}^{-1}$; Coziol et al. 2009; Lauer et al. 2014), suggesting that these objects may not reside at the bottom of the cluster potential well.

Recent work (e.g., Rhee et al., 2017; Gozaliasl et al., 2020) combines the cluster-centric velocities and cluster-centric radii in a single diagram. This phase-space diagram can be used to extract information about the assembly history of clusters (Mahajan et al., 2011; Hernández-Fernández et al., 2014, e.g.). For example, one expects recently accreted galaxies to show higher relative velocities and offsets from the centre than objects accreted at an earlier time. Objects accreted early are usually found within the core of the virialised region and show a small velocity spread (Noble et al., 2016; Gozaliasl et al., 2020).

In this paper, we investigate the kinematics of the hosts of radio galaxies in groups (BGGs and 'satellites ${ }^{1}$ ), comparing them to the kinematics of galaxies with no detected radio emission. To this end, we rely on a recently published sample of X-ray galaxy groups (Gozaliasl et al., 2019), combining

1 Throughout this work, we will refer to non-BGGs as satellites for easier reading it with optical, kinematic and spectroscopic data. All host groups were identified in the 2 square degree COSMOS field, with a mass range of $M_{200}=8 \times 10^{12}-3 \times 10^{14} M_{\odot}$, where the upper limit of this range corresponds to a virial temperature of $\sim 4 \mathrm{keV}$.

This paper is structured as follows: In Sec. 2 we describe our sample and how we compiled it. In Sec. 3.1 we perform a phase-space analysis and in Sec. 3.2, we compare it to cosmological simulations. In Sec. 3.3 we explore the properties of our sample and derive scaling relations in Sec. 3.4. In Sec. 3.5 we discuss implications for AGN feedback before we conclude in Sec. 4.

Throughout the paper, we assume a standard $\Lambda$ CDM cosmology with $\mathrm{H}_{0}=70 \mathrm{~km} \mathrm{~s}^{-1} \mathrm{Mpc}^{-1}, \Omega_{\Lambda}=0.73$ and $\Omega_{\mathrm{M}}=1-\Omega_{\Lambda}=0.27$.

\section{THE SAMPLE}

Gozaliasl et al. (2019) presented a sample of 247 X-ray selected galaxy groups in the 2 square degree COSMOS field at a redshift range of $0.08 \leq z<1.53$. The same sample was also studied in Pasini et al. (2020) making use of radio data from the VLA-COSMOS survey (Schinnerer et al., 2010) and of new MeerKAT observations that are part of the MIGHTEE survey (Jarvis et al., 2016). In this paper, they found evidence for a correlation between the X-ray luminosity of galaxy groups and the radio luminosity of the central AGN (see Pasini et al. 2020 for further details).

Before we can perform a dynamical analysis, we need to determine cluster membership through spectroscopic redshifts. To this end, we have vetted the group membership catalog of Gozaliasl et al. (2019) by applying the CLEAN algorithm of Mamon et al. (2013), which removes the galaxies exceeding the escape velocity of the group as a function of clustercentric radius from the group. We kept groups with more than four member galaxies and removed all of those galaxies that have no spectroscopic data. This resulted in a total of 79 groups, with 998 member galaxies, which limits our study to redshifts of $z \leq 1.0$. Among these members, we identified 70 BGGs, that were found to be the most massive in each group by Gozaliasl et al. (2014, 2019). We have computed the gapper velocity dispersion estimates $\sigma_{\mathrm{v}}$ following Beers et al. (1990).

If the most massive galaxy only has photometric redshifts, it gets excluded from our sample. As a result, not every group has its BGG included in this work, and for some groups the BGG identification could even be wrong if another galaxy was mistakenly identified as BGG. Therefore, we performed a further check and found this to be the case only in one group, where the galaxy previously classified as BGG was actually a satellite.

The spectroscopic member galaxies thus obtained were then matched to the VLA-COSMOS Deep survey at $1.4 \mathrm{GHz}$ $\left(\mathrm{rms} \sim 12 \mu \mathrm{Jy}_{\text {beam }}{ }^{-1}\right.$, beam $=2.5^{\prime \prime} \times 2.5^{\prime \prime}$, Schinnerer et al. 2010). The cross-match was performed by assuming an association between radio emission and optical galaxy when their angular distance is less than the width of the beam in the VLA-COSMOS survey. We found that 50 groups host at least 1 radio source according to our criteria, with a total of 79 detected radio galaxies (28 in BGGs and 51 in satellites). 19 of these groups host more than one radio galaxy, while for 


\begin{tabular}{ccc}
\hline \hline & BGGs & Satellites \\
\hline Radio detection & 28 & 51 \\
No radio detection & $42^{a}$ & $877^{b}$ \\
\hline Total & 70 & 928 \\
\hline
\end{tabular}

Table 1. Properties of the samples studied in this work.

${ }^{a}: 19$ from the 50 groups with at least one radio galaxy detected and 23 from the remaining 29 groups.

${ }^{b}: 664$ from the 50 groups with at least one radio galaxy detected and 213 from the remaining 29 groups.

31 we only detect one source. Groups with no detected radio emission are therefore 29, with 236 member galaxies in total (23 BGGs and 213 satellites). The remaining 683 galaxies (19 BGGs and 664 satellites) with no radio emission belong to the 50 groups with at least one radio galaxy. The characteristics of the samples are briefly summarized in Table 1.

The temperature of the groups was determined through the $T_{X}-\mathrm{L}_{X}$ scaling relation (Giles et al., 2016; Kettula et al., 2015). A small subsample also had a direct measurement of the temperature available (Kettula et al., 2013). We find that these measurements are consistent with the scaling relation above.

The final catalog presents a set of multi-wavelength observables for each group (X-ray luminosity, temperature), for the member galaxies (spectroscopic redshift, velocity dispersion, proper velocity, stellar mass) and for the corresponding radio source, when present (1.4 GHz power, Largest Linear Size). A selection bias could be introduced by those galaxies - and therefore the host groups - that do not show radio emission according to our criteria. The reason for this could be the lack of an AGN, or limitations set by the sensitivity of VLACOSMOS. Among all observable galaxies, Padovani et al. (2017) claim that around $\sim 1 \%$ host an AGN. Nevertheless, this value should increase in overdense environments such as galaxy groups. Sabater et al. (2019) report a 100\% detection rate for galaxies with $\mathrm{M}>10^{11} \mathrm{M}_{\odot}$, but this fraction has been observed to strongly vary depending on the host galaxy stellar mass (Kauffmann et al., 2003). Among our sample, $8 \%$ host an AGN. The radio luminosity distribution for all galaxies with detected radio emission is shown in Fig. 1.

The luminosity distributions span the range $L_{R} \sim 2 \times 10^{21}$ $\mathrm{W} \mathrm{Hz}{ }^{-1}-L_{R} \sim 3 \times 10^{25} \mathrm{~W} \mathrm{~Hz}^{-1}$. The end of the range is lower than the BCG luminosity distribution of Hogan et al. (2015) that reaches $L_{R} \sim 10^{27} \mathrm{~W} \mathrm{~Hz}^{-1}$. The reason for this is that we are only considering galaxy groups, where the radio power of the central galaxy is generally lower than for clusters. This was also found in Pasini et al. (2020) by investigating the same parent sample used for this work, composed by 247 COSMOS galaxy groups. In Pasini et al. (2020), the luminosity reaches $\sim 10^{27} \mathrm{~W} \mathrm{~Hz}^{-1}$ in a small ( $<5$ out of 247 groups) number of outliers that show very extended AGN emission. The reason why these sources are excluded from the present work is that no spectroscopic data is available for the optical host. Our analysis will therefore be limited to $L_{R}<10^{26} \mathrm{~W} \mathrm{~Hz}^{-1}$.

At the highest redshift of the sample $(z \sim 0.98)$, the VLACOSMOS sensitivity at $3 \sigma$ corresponds to $L_{1.4} \mathrm{GHz} \sim 2 \times 10^{23}$ $\mathrm{W} \mathrm{Hz}{ }^{-1}$. With the mean redshift setting around $z \sim 0.4$, we should be able to pick most of the radio sources brighter than

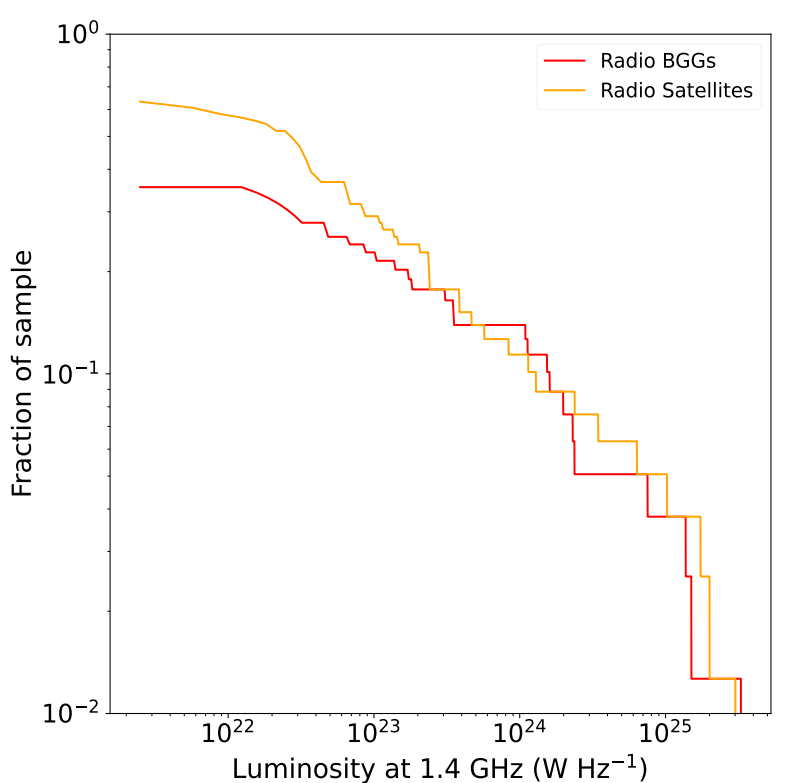

Figure 1. Cumulative $1.4 \mathrm{GHz}$ luminosity distribution for all 79 galaxies with radio emission, divided into BGGs (red) and satellites (orange). The $y$-axis reflects the relative fractions of BGGs (28 out of 79 galaxies) and satellites (51 out of 79 galaxies) that compose the radio galaxy sample.

$10^{22} \mathrm{~W} \mathrm{~Hz}^{-1}$ at $1.4 \mathrm{GHz}$. This yields a sample that is representative of the radio luminosity function usually observed for radio galaxies (e.g. Hogan et al., 2015). Therefore, undetected radio emission should not affect our analysis, which focuses on the comparison between BGGs with detected radio emission (hereafter radio BGGs), satellites with detected radio emission (hereafter radio satellites), BGGs with no radio emission and satellites with no radio emission.

\section{RESULTS AND DISCUSSION}

\subsection{Phase-space analysis}

We performed a phase-space analysis by comparing the cluster/group-centric velocity with the cluster/group-centric offset of the hosted galaxies. This diagram conveys information about the assembly and accretion history of these objects. In the left panel of Fig. 2 we show the phase-space diagram for radio BGGs, radio satellites, non-radio BGGs and non-radio satellites. Following Rhee et al. (2017) and Gozaliasl et al. (2020), the position of each object in this diagram is an indicator of the infall time $\left(t_{\text {inf }}\right)$ of the galaxy, with ancient infallers $\left(6.45 \mathrm{Gyr}<t_{\mathrm{inf}}<13.7 \mathrm{Gyr}\right)$ found to the left of the black dotted line in Fig. 2, while intermediate infallers $\left(3.63 \mathrm{Gyr}<t_{\text {inf }}<6.45 \mathrm{Gyr}\right)$ cover the whole offset range below the grey dashed curve. Galaxies above the green line are classified as recent infallers, while the remaining ones cannot be attributed to any of these classes. This does not affect the following analysis since our purpose is to distinguish ancient infallers from all the other objects. Out of 28 radio BGGs, only $5(\sim 18 \%)$ are not classified as ancient infallers, which 

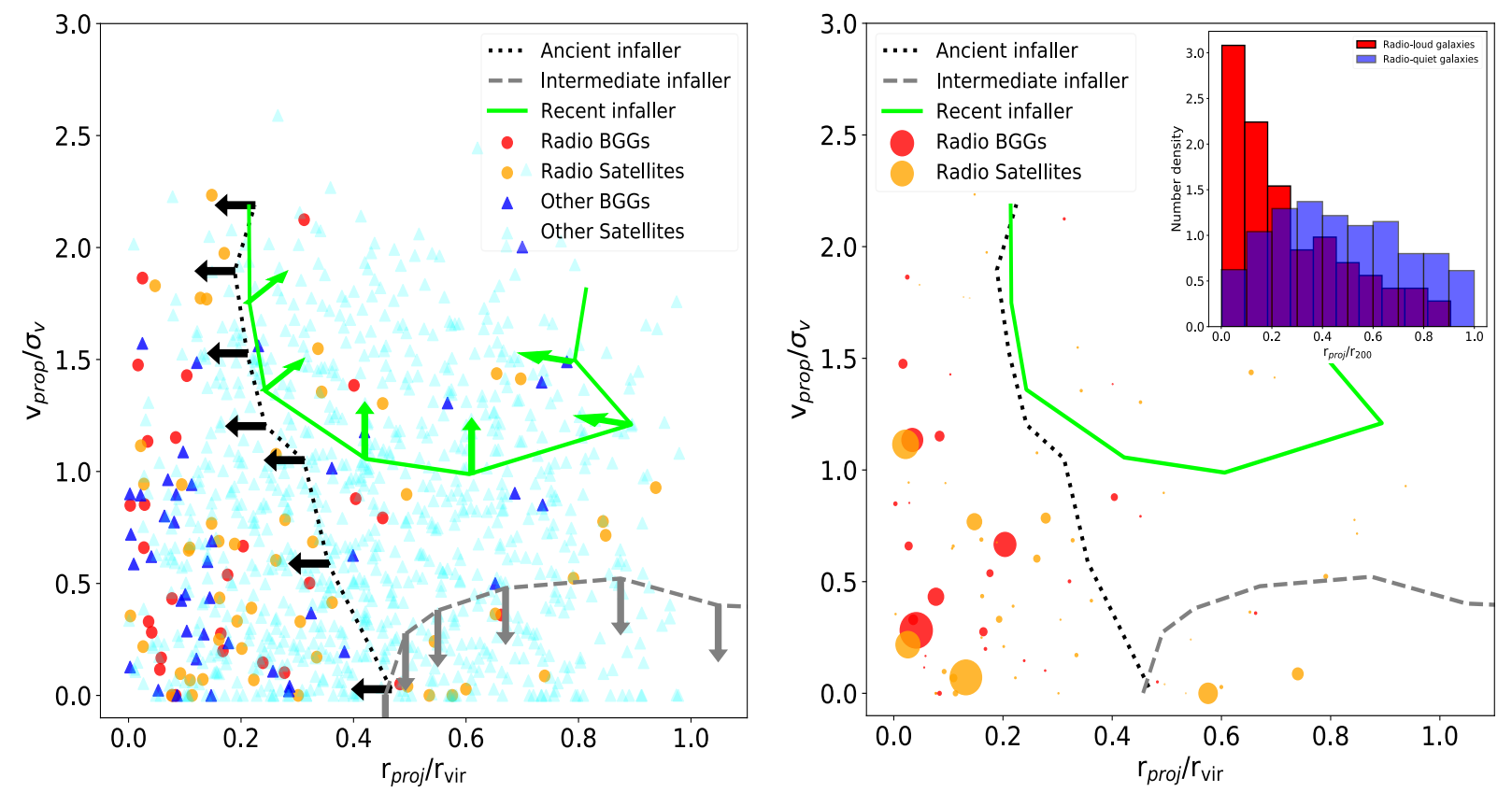

Figure 2. Left: Phase-space diagram for radio BGGs (red), non-radio BGGs (blue), radio satellites (orange) and non-radio satellites (cyan). The $x$-axis represents the ratio between the distance from the group centre and $R_{\text {vir }}$, while the $y$-axis is the ratio between the line-of-sight velocity and the (one-dimensional) velocity dispersion. The different regions in the diagram indicate ancient infallers (left of black dotted line), intermediate infallers (below the grey dashed line) and recent infallers (above the green line). Right: Phase-space diagram restricted to radio BGGs and radio satellites only, with the points sized for the power of the corresponding radio galaxy. The top-right histogram shows the offset distribution for galaxies with (red) and without (blue) radio emission.

constitute $\sim 82 \%$ of the sample. On the other hand, the sample of 42 non-radio BGGs is composed of 33 ancient infallers ( $\sim 78 \%)$, consistently with Gozaliasl et al. (2019), who also found that BGGs are mostly ancient infallers. The sample of radio satellites show $\sim 65 \%$ ancient infallers. Finally, only $\sim 41 \%$ of non-radio satellites present this classification.

The right panel of Fig. 2 shows objects with radio emission only (BGGs and satellites), with the size of the symbols proportional to the power of the radio source. The top-right histogram shows the offset distribution for galaxies with and without radio emission. The comparison between the radio and non-radio samples in the histogram clearly indicates that most of the galaxies with radio emission are ancient infallers (56 out of $79, \sim 71 \%$ ), strongly peaking at low offsets, while the distribution of the distances from the group centre for galaxies with no radio emission is more uniform across $R_{\text {vir }}$. The phase-space analysis applied to radio objects-only also suggests that powerful radio galaxies $\left(L_{R}>10^{23} \mathrm{~W} \mathrm{~Hz}^{-1}\right)$ are always located close to the group centre $\left(<0.2 R_{\mathrm{vir}}\right)$. This is expected since central galaxies switch the SMBH on much easily. The gas cooled out of the Intra-Group Medium (IGrM) can feed the AGN if the galaxy lies close to the group density peak, where the cooling is more efficient. Nevertheless, galaxies located in the outskirts or outside the cooling radius of the group can still show radio emission. However, they might have to rely on more episodic triggers, such as mergers or interactions with other objects. Only in a few cases, their radio power is able to become comparable to those of central galaxies. This happens especially because the low density in the outskirts of galaxy groups sometimes allow them to grow rapidly in size (see also Pasini et al. 2020).
A further consequence of this is that radio BGGs have a higher chance than non-radio BGGs to lie close to the group centre. This is particularly true for the most powerful ones $\left(L_{R}>10^{23} \mathrm{~W} \mathrm{~Hz}^{-1}\right)$, that in our samples always lie within $0.2 R_{\text {vir }}$. Therefore, the detection of a powerful radio source in a group can help identify the group centre. Finally, it is worth noting that no difference is visible in terms of velocity ratio between powerful BGGs and those with $L_{R}<10^{23} \mathrm{~W}$ $\mathrm{Hz}^{-1}$.

\subsection{Comparison with cosmological simulations}

Here, we compare our samples with the theoretical predictions from the HORIZON-AGN (HZ) simulation ${ }^{2}$ (Dubois et al., 2014). The HORIZON-AGN simulation is a cosmological hydrodynamical simulation of $100 \mathrm{Mpc} / h$ comoving box containing $1024^{3}$ Dark Matter particles. The simulation is performed with the adaptive-mesh refinement code RAMSES (Teyssier, 2002) including gas dynamics, gas cooling and heating, and sub-grid models for star formation, stellar and AGN feedback. The AdaptaHOP halo finder (Aubert et al., 2004) was run on both the stellar and DM particle distributions to identify galaxies and halos (see Laigle et al. 2019 and Gozaliasl et al. 2019 for further details). Each galaxy is then associated with its closest main halo. To match the observational definition, the BGG is identified as the most massive galaxy within the virial radius of the main halo.

The left panel of Fig. 3 shows the probability density distribution of the velocity ratio for radio and non-radio BGGs

${ }^{2}$ https://www.horizon-simulation.org 

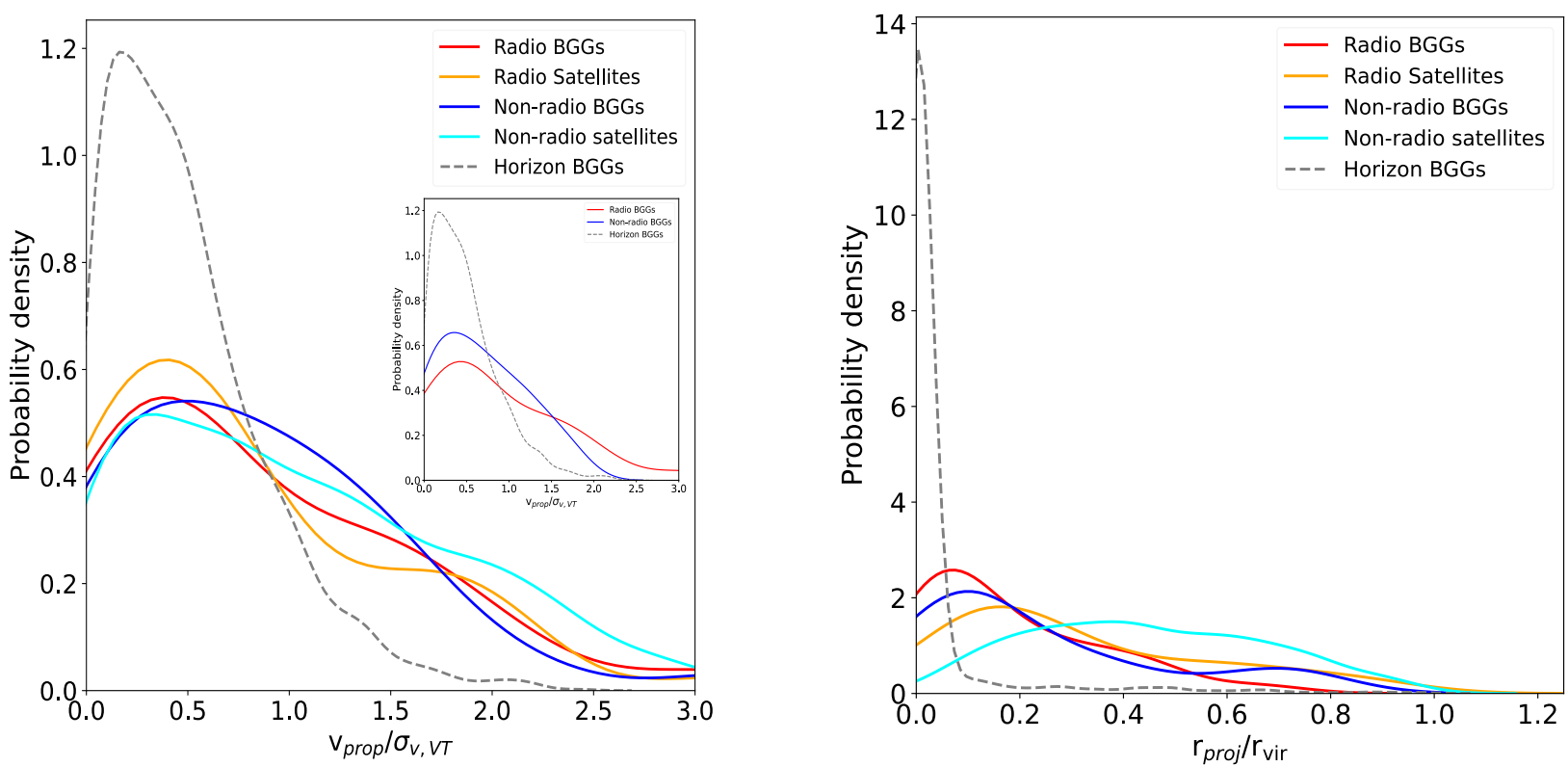

Figure 3. Left: Probability density (obtained through a gaussian kernel density estimate) vs. velocity ratio for radio and non-radio BGGs (red and blue), for radio and non-radio satellites (orange and cyan) and for HZ-simulated BGGs (dashed gray).The subplot shows the same distribution restricted to only BGGs with offset $<0.3 R_{\mathrm{vir}}$. Right: Probability density vs. offset from the group centre for radio and non-radio BGGs (red and blue), for radio and non-radio satellites (orange and cyan) and for HZ-simulated BGGs (dashed gray).

and for radio and non-radio satellites. Here, instead of the gapper velocity dispersion $\sigma_{\mathrm{v}}$ exploited in Fig. 2, we use the velocity dispersion estimated from X-ray emission $\sigma_{\mathrm{v}, V T}$ (see below). The gray dashed curve represents the distribution for simulated BGGs obtained by HZ-AGN, whose mass and redshift evolution were already studied and compared to COSMOS BGGs and satellites in Gozaliasl et al. (2020). Here, our purpose is to understand whether the dynamical properties of our four samples differ significantly from each other, and how they compare to simulated galaxies.

The distributions of radio and non-radio BGGs peak at $v_{\text {prop }} / \sigma_{\mathrm{v}, \mathrm{VT}} \sim 0.4$ and 0.5 , respectively. For radio BGGs, the mean velocity ratio is $\sim 0.84$ and the median velocity ratio is $\sim 0.63$. For non-radio BGGs the mean velocity ratio is $\sim$ 0.85 and the median is $\sim 0.78$. Radio satellites have the peak velocity ratio around $\sim 0.45$, with a mean of $\sim 0.78$ and a median of $\sim 0.59$. The distribution for non-radio satellites is broader, setting the peak at $\sim 0.3$ but becoming the dominant sources at $v_{\text {prop }} / \sigma_{\mathrm{v}, \mathrm{VT}} \geq 1.6$, with a mean of $\sim 1.04$ and a median of $\sim 0.89$. Finally, BGGs in the HZ-AGN simulation are strongly peaked around $v_{\text {prop }} / \sigma_{\mathrm{v}, \mathrm{VT}} \sim 0.2$, with a mean of $\sim 0.49$ and a median of $\sim 0.41$.

Given that the simulation represents the dynamics of the central galaxy, in the subplot of Fig. 3 we select only BGGs within $0.3 R_{\text {vir }}$ from the X-ray center, to see if this changes the observed displacement of the curve with respect to HZ BGGs. We see no difference in the distribution for radio BGGs, while the curve for non-radio BGGs becomes tighter. Nevertheless, the distribution for HZ BGGs remains much more strongly peaked at low velocity with respect to real BGGs, with a steeper decrease after the peak.
The probability distribution of offsets from the group centre, shown in the right panel of Fig. 3, confirms that BGGs (radio, non-radio and simulated) are highly concentrated within $0.2 R_{\mathrm{vir}}$. At higher radii the curves of radio and nonradio BGGs decrease in a similar fashion, while the probability density of simulated BGGs already steepens at $\sim 0.1 R_{\mathrm{vir}}$. Nevertheless, in simulations BGGs tend to be more massive than their observational counterparts (e.g., Bahé et al., 2017; Henden et al., 2019; Bassini et al., 2020). For this reason, they are closer to the centre and exhibit less spread in velocity. On the other hand, satellites exhibit a broader distribution, with radio satellites prevailing at offsets $\leq 0.3$, while at outer radii non-radio satellites become dominant.

All BGGs were observed as a part of zCOSMOS survey (Lilly et al., 2007), with a redshift error of $\sim 55 \mathrm{~km} / \mathrm{s}$. Missing objects were covered by FORS2 program (George et al., 2011 ), with a similar redshift precision, and at $z>0.7$ by GEEC2 (Balogh et al., 2011), with a redshift precision of $80 \mathrm{~km} / \mathrm{s}$. Based on the work of Saro et al. (2013), the uncertainty of velocity dispersion measurement is high with typical number of spectroscopic members of COSMOS X-ray galaxy groups. Better constraints on the velocity dispersion are obtained using scaling relations of $L_{X}-M_{200}$ (Leauthaud et al., 2010) and $M_{200}-\sigma_{\mathrm{v}, \mathrm{VT}}$ (Carlberg et al., 1997). The lognormal scatter $L_{X}-\sigma_{\mathrm{v}, \mathrm{VT}}$ relation is measured to be 0.13 by Kirkpatrick et al. (2021). Using the velocity dispersion from scaling relations, the disagreement with simulations consists in a wider tail above $0.7 \sigma$ extending to $2.5 \sigma$. The spread due to uncertainty in the mean redshift is typically $0.3 \sigma_{\mathrm{v}}, \mathrm{VT}$ and always better than $0.45 \sigma_{\mathrm{v}, \mathrm{VT}}$ and cannot explain the large tail. 
Positional displacement of BGGs from the center of the halo is constrained to be within $0.1 R_{\text {vir }}$ in simulations, while our data shows much broader range of offsets between BGG and X-ray peak. George et al. (2012) found that BGGs in the vicinity of X-ray centers (within $0.25 R_{\text {vir }}$ ) are good tracers of projected mass centers, with an offset less than $0.1 R_{\mathrm{vir}}$, but BGGs with strong offsets from X-ray center do not trace the center of mass and the corresponding mass profiles suggest merging. Thus, the broad distribution of offsets within 0.3 $R_{\text {vir }}$ is due to displacements of X-ray peak, while larger offsets are merger driven. For the offset peak of HZ galaxies to match that of our data, it would require a systematic shift of $\sim 0.1$ $R_{\text {vir }}\left(10^{\prime \prime}-6^{\prime}\right)$.

To quantify our results, we performed a KolmogorovSmirnov (KS) test to compare the radio and non-radio BGG distributions with simulated BGGs. Our null-hypothesis is that the samples are drawn from the same parent distribution. The KS-test on the phase-space distributions of radio BGGs and simulated BGGs gives a null-hypothesis probability of $p=1.6 \times 10^{-13}$, while the comparison between nonradio BGGs and simulated BGGs results in $p=3.1 \times 10^{-17}$. This suggests that the simulation is not able to reproduce our samples, indicating that it may need additional physics to reproduce the true population of BGGs.

\subsection{Properties of the samples}

The left panel of Fig. 4 shows the stellar mass distribution for the four samples: radio BGGs, radio satellites, non-radio BGGs and non-radio satellites. Non-radio satellites dominate the low-mass regime, from $10^{9} \mathrm{M}_{\odot}$ to $5 \times 10^{10} \mathrm{M}_{\odot}$. Lowmass radio BGGs start to appear around $3 \times 10^{10} \mathrm{M}_{\odot}$, while non-radio BGGs go down to $10^{10} \mathrm{M}_{\odot}$. Radio BGGs become dominant for stellar masses $\geq 3 \times 10^{11} \mathrm{M}_{\odot}$. In order to compare the mass distributions of the BGG samples, we show the histograms of radio and non-radio BGGs in the right panel of Fig. 4. Out of 28 radio BGGs, 27 have $M_{*}>10^{11}$ $\mathrm{M}_{\odot}(\sim 96 \%)$, while the same fraction for non-radio BGGs is $\sim 83 \%$ (35 out of 42 ). The small size of the samples does not lead to statistically significant results, but radio BGGs trend towards higher masses, while it is less likely for a BGG with $M_{*}<10^{11} \mathrm{M}_{\odot}$ to host a radio galaxy.

This is even more significant when considering that massive BGGs are found in more massive groups and vice versa, as discussed in several papers (e.g., Stott et al., 2010; Gozaliasl et al., 2016) and shown in Fig. 5. This is in agreement with Gaspari et al. (2019) who observed that more massive SMBHs correlate with larger and hotter X-ray halos. The same is also found in cosmological simulations by, e.g., Bassini et al. (2019); Truong et al. (2021). In combination with the result shown above that radio BGGs are usually more massive than those with no radio emission, this suggests that radio BGGs are more likely to be hosted in high-mass groups. Since radio-BGGs statistically exhibit smaller offsets from the centre than non-radio BGGs (see Sec. 3.1), we can argue that this could affect the trigger of the AGN. Indeed, Fig. 2 shows that a number of non-radio BGGs have large offsets. This is not surprising considering that the group centre as we defined it, i.e., the bottom of the potential well, is where the hot gas density is higher and cooling is faster, especially in more relaxed systems. Stronger cooling implies more condensing mass (Gaspari et al. 2019) which feeds the supermassive black hole (SMBH) (e.g., see the GR-rMHD simulations by Sạdowski \& Gaspari 2017). Nevertheless, there are also highmass non-radio BGGs which do not host a radio source, at least with our sensitivity limit. This could likely be explained by the flickering duty cycle involved in the AGN feeding and feedback self-regulation (Sec. 3.5) .

In order to determine whether one of our samples shows any divergence from standard scaling relations, we investigated the correlation between X-ray luminosity and observed velocity dispersion $\left(\sigma_{\mathrm{v}}\right)$ for groups hosting radio and nonradio BGGs, plotted in Fig. 6 (e.g., Wu et al., 1999; Mahdavi \& Geller, 2001; Zhang et al., 2011; Gozaliasl et al., 2020). None of the samples seem to show any deviation and both follow the same correlation. This is confirmed by the KS test $(p=0.06)$, which suggests that the two distributions are similar and that no discernible difference exists in the $\sigma_{\mathrm{v}}-\mathrm{L}_{X}$ correlation between the groups hosting radio and non-radio BGGs.

We also looked for evidence of recent interactions with other galaxy groups by inspecting the Chandra and XMMNewton observations used to build the original galaxy groups catalog (Gozaliasl et al., 2019). We find that only for one group it is possible to detect hints of mergers (LSS 17, see Smolčić et al. 2007). The images are too shallow to reveal anything for the other objects. We will return to this issue in Sec. 3.5, but deeper X-ray observations will be needed for this purpose.

It is well-known that the magnitude difference between the first- and second-rank galaxies in a group/cluster is helpful to trace their merger history and evolution (Ponman et al., 1994; Gozaliasl et al., 2014, 2019). Simulations have shown that mergers in galaxy groups lead to runaway growth of the BGG (e.g., Cavaliere et al., 1986; Mamon, 1992), at the expense of the second brightest galaxy. Therefore, the magnitude gap between the BGG and the second-rank galaxy should increase in time, finally leading to a situation in which the central, elliptical BGG, lying in an X-ray luminous halo, is surrounded by faint satellites. These groups are known as fossil groups (Jones et al., 2003). Therefore, one expects larger gaps in more relaxed groups, where the offset between the BGG and the halo centre is lower. The magnitude gap - offset relation for our parent sample of galaxy groups has already been studied in Gozaliasl et al. (2019). Here, we wish to understand how this gap relates to BGGs with and without radio emission.

In Fig. 7, we show the R-band magnitude gap distribution for our brightest groups galaxies, classified into radio and non-radio BGGs. The gap was measured within $0.5 R_{200}$, following (Jones et al., 2003). The second-rank galaxy of each group was picked, regardless of it having spectroscopic or photometric identification, using the full membership catalog studied in Gozaliasl et al. (2020). Both distributions peak around $\sim 1$, with a clear concentration of objects before $\sim 1.5$. No clear difference in the trend is detected between radio and non-radio BGGs, as also confirmed by the KS test $(p=0.13)$. Nevertheless, our catalogs only have a few tens of objects, and analyses on larger samples could help to address how the magnitude gap relates to radio and non-radio BGGs.

\subsection{Scaling relations}

Pasini et al. (2020) explored a correlation between the 1.4 

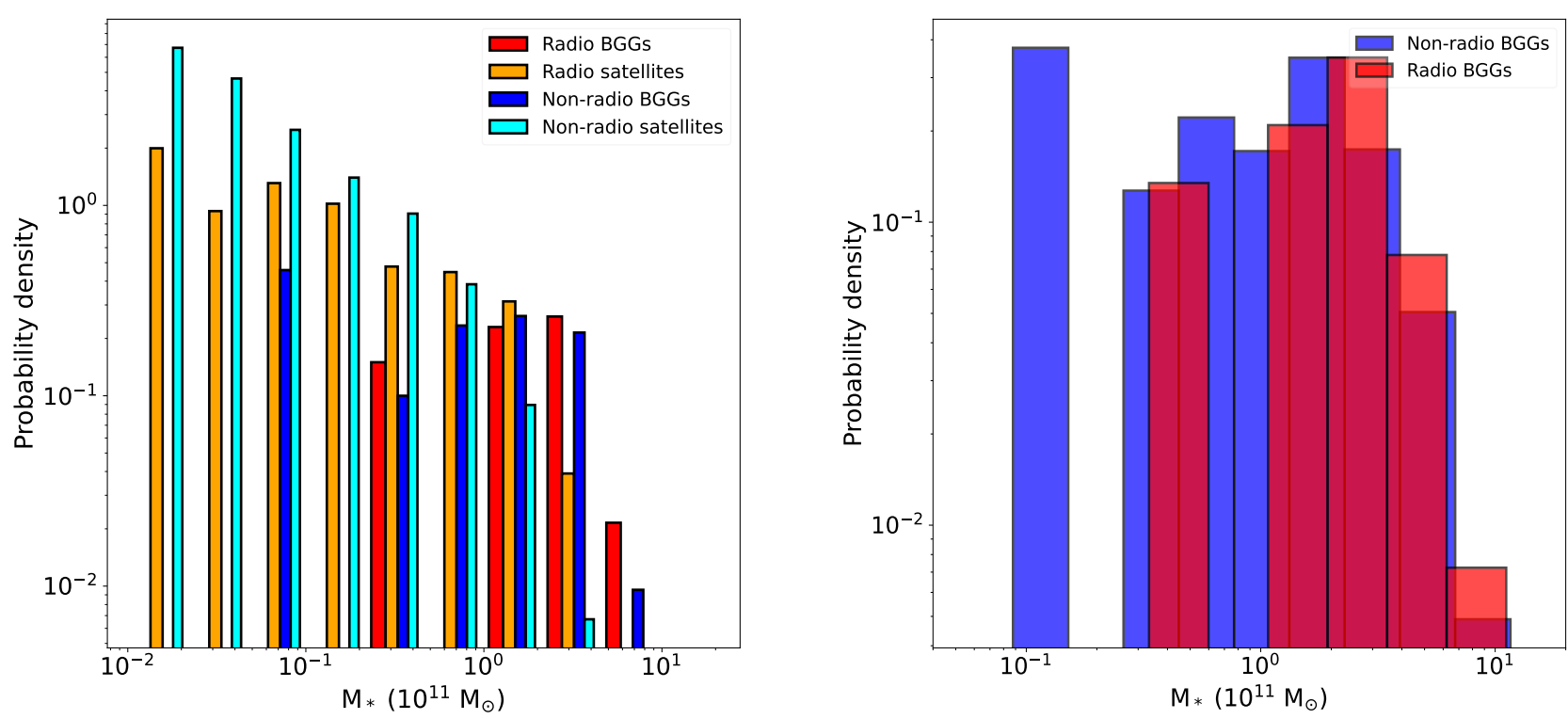

Figure 4. Left: Stellar mass distribution for radio (red) and non-radio (blue) BGGs, and for radio (orange) and non-radio (cyan) satellites. Right: Stellar mass distribution restricted to radio (red) and non-radio (blue) BGGs.

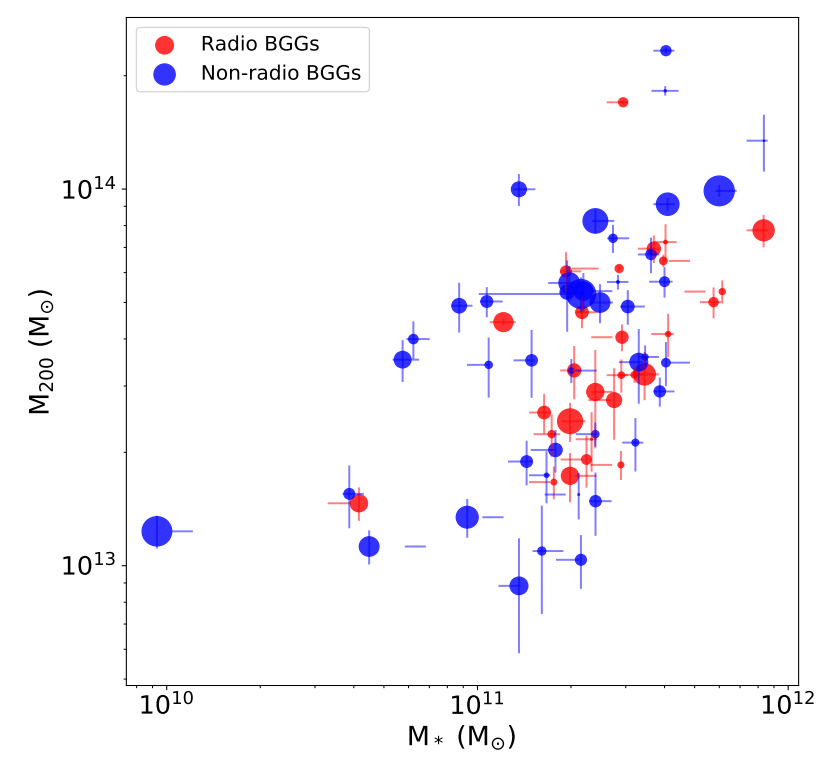

Figure 5. Galaxy groups $M_{200}$ vs. stellar mass for the radio-BGG (red) and non-radio BGG (blue) samples. The size denotes the distance of the BGG from the group centre, with bigger points indicating larger offsets.

GHz radio power of the central AGN and the X-ray luminosity of the host galaxy group. Since the sample of radio BGGs studied in this work is derived from the same parent catalog of X-ray groups (Gozaliasl et al., 2019), we expect to find a similar correlation, which is shown in the left panel of Fig. 8.

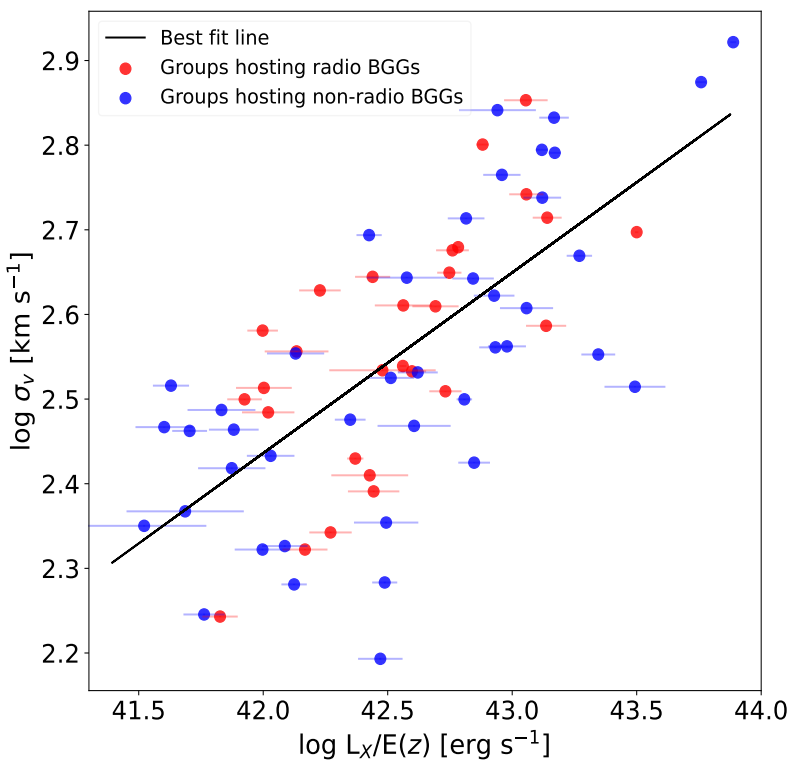

Figure 6. Observed velocity dispersion of the host group vs. X-ray luminosity for radio (red) and non-radio (blue) BGGs. The black line represents the scaling relation obtained by computing the $\mathrm{L}_{X}$ - M and the M - $\sigma_{v, V T}$ correlations presented in Leauthaud et al. (2010) and Mamon et al. (2013), respectively.

The sample was divided into relaxed objects (offset < $0.2 R_{\text {vir }}$ and $v<0.5 v_{\text {disp }}$ ), high-velocity objects (offset $<0.2 R_{\text {vir }}$ and $v>0.5 v_{\text {disp }}$ ) and offset objects (offset 


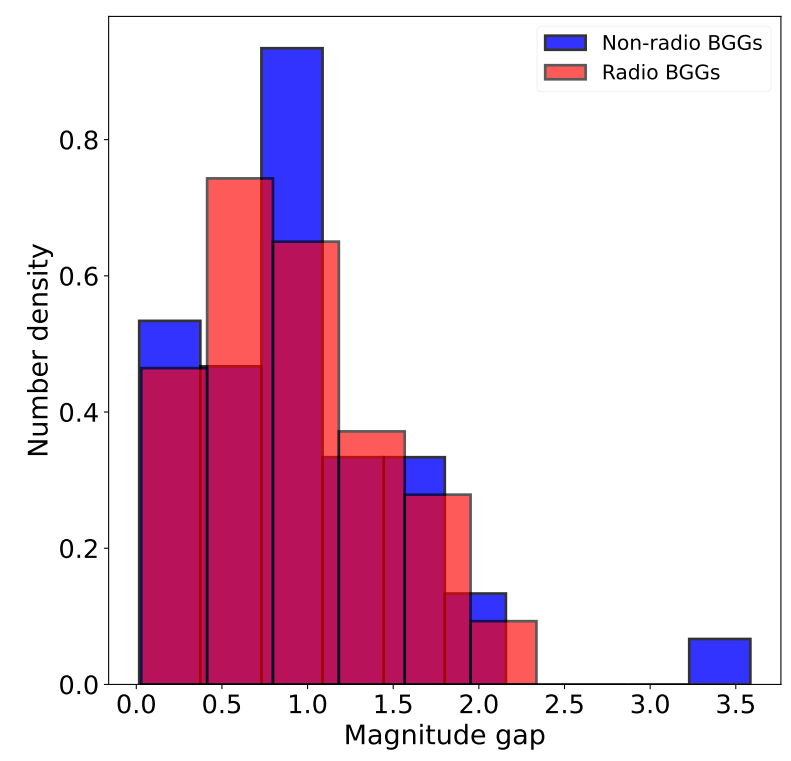

Figure 7. Magnitude gaps (R-band) of radio (red) and non-radio (blue) BGGs from the corresponding second-rank galaxy.

$\left.>0.2 R_{\text {vir }}\right)$. We find no significant differences between the three subsamples. We then applied Bayesian inference to extract the best-fit relation, by using $\operatorname{linmix}^{3}$ (cf. Sec. 2.2 in Gaspari et al. 2019 for a discussion of its features). A linear fit in $\log -\log$ scale was performed in the form:

$Y=\alpha+\beta X+\epsilon$,

with $\alpha$ and $\beta$ representing the intercept and the slope, respectively, while $\epsilon$ is the intrinsic scatter of the relation. For the AGN power versus X-ray luminosity correlation, we find $\alpha=-9.53 \pm 18.19, \beta=0.94 \pm 0.43$ and $\epsilon=0.96 \pm 0.31$. As expected, this estimate is consistent with the slope of $L_{R} \propto(1.07 \pm 0.12) L_{X}$ presented in Pasini et al. (2020), obtained through least-squares linear regression. However, we note that the errors are large due to the small sample size. It is generally understood that the X-ray emission by clusters and groups is tightly linked to the temperature of the ICM (Lovisari et al., 2020). Therefore, if a correlation of the hot plasma with the AGN exists, we expect a similar link with the gas temperature, too. The right panel of Fig. 8 shows the correlation between the $1.4 \mathrm{GHz}$ AGN power and the $\mathrm{X}$-ray temperature of the intragroup medium of the host. Again, no difference is apparent among the subsamples. We find $\alpha=30.57 \pm 0.19$ and $\beta=2.35 \pm 1.25$, with intrinsic scatter $\epsilon=1.01 \pm 0.32$.

The significant positive and steep correlation with X-ray halo properties can be compared with those by Gaspari et al. (2019), who found also key positive X-ray halo correlations between the (direct/dynamical) SMBH masses and the observed $T_{\mathrm{x}}$ and $L_{\mathrm{x}}$. Specifically, their group-dominated sample

3 https://github.com/jmeyers314/linmix. show a slope $M_{\mathrm{BH}} \propto T_{\mathrm{x}}^{2.14 \pm 0.25}$, which is well consistent with the above mean relation. This suggests that, despite the AGN power being an instantaneous measure $\left(L_{\mathrm{R}} \sim P_{\mathrm{BH}} \propto \dot{M}_{\mathrm{BH}}\right)$, the mean $L_{\mathrm{R}}-T_{\mathrm{x}}$ is not drastically altered by the details of the feedback duty cycle, except by introducing a larger intrinsic scatter $(4 \times)$ due to the chaotic intermittency. Such a variable duty cycle is a feature corroborated by a wide range of numerical and observational studies (e.g., McNamara \& Nulsen 2007; Gaspari et al. 2011; Fabian 2012; Prasad et al. 2015; Yang \& Reynolds 2016). The $L_{\mathrm{R}}-L_{\mathrm{x}}$ relation appears to be slightly steeper than the $M_{\mathrm{BH}}-L_{\mathrm{x}}$, although still comparable within the 1- $\sigma$ uncertainty. Overall, as we will discuss in Sec. 3.5, a hotter halo implies a larger gas mass, stronger CCA feeding and stronger AGN feedback power, thus establishing major positive correlations. In this regard, the $L_{\mathrm{R}}-T_{\mathrm{x}}$ relation can be used as a proxy to describe AGN feedback and feeding rates.

In passing, it is worth noting that all BGGs found at distances of more than $0.2 R_{\text {vir }}$ from the X-ray centre (green) lie underneath the mean best-fit line suggesting that, at a given $\mathrm{X}$-ray luminosity (or temperature), their radio luminosity is lower than in the more central BGGs. Again, the centeredness of the system appears to be key to initiate stronger feedback (and related feeding; see next Sec. 3.5).

\subsection{AGN feeding and feedback cycle}

In the previous sections, we showed that AGN activity is usually detected at the centres of galaxy groups, regardless of the properties of the optical galaxy. The triggering of the (mechanical) AGN activity thus appears to depend on the position of the host halo. In CCA, the central position in the group promotes the condensation and inflow of low-momentum gas from both the internal galactic gas and external intragroup medium. As a consequence, in CCA the AGN radio power correlates with the X-ray halo temperature (and luminosity), consistent with the results in Sec. 3.4. During CCA, inelastic collisions between the condensed clouds and filaments drive a rapid inflow toward the micro scale (a few tens of the Schwarzschild radius; Gaspari \& Sądowski 2017). The ultrafast outflows and jets then entrain gas at the meso-scale $(\sim 1 \mathrm{kpc})$, with their kinetic energy dissipated and released at the macro-scale (tens $\mathrm{kpc}$ ) via X-ray bubbles, shocks, and turbulence (e.g., Gaspari et al. 2011; Barai et al. 2016; Yang et al. 2019; Liu et al. 2019; Wittor \& Gaspari 2020). Thus, the ensuing cooling flow of the central intragroup medium can be quenched rapidly by AGN heating, leading to a new feedback cycle (Gaspari et al. 2020 for a review and unification diagram of such processes). On the other hand, the AGN in non-centrals/'satellites' can only feed from the diffuse gas inside its host galaxy ('internal weather'), and cannot easily tap into the reservoir of the intragroup medium due to their large infall/relative velocity. This is supported by our results, as powerful radio galaxies mostly lie at the group centre.

An alternative accretion mode for the SMBH is hot accretion. This mode can take various forms, from pure Bondi accretion - usually based on idealized assumptions such as the presence of a spherically symmetric, steady, adiabatic and gaseous atmosphere (Bondi, 1952) - to Advection-Dominated Accretion Flow (ADAF, Narayan \& Yi 1995). Unlike in CCA, in hot-mode accretion, the gravitational pull of the $\mathrm{SMBH}$ 

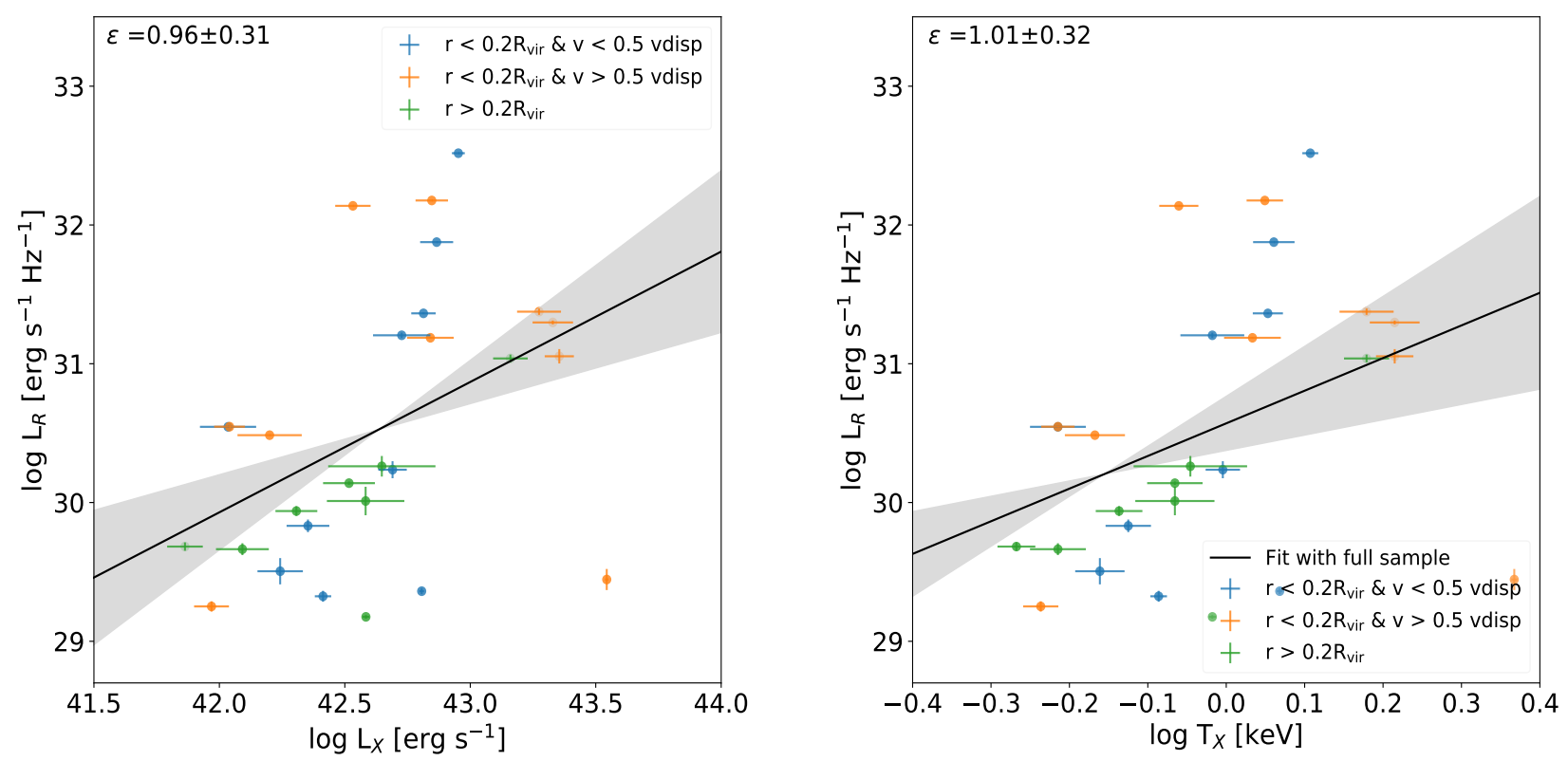

Figure 8. Left: $1.4 \mathrm{GHz}$ AGN radio power vs. X-ray luminosity of the host group for the radio BGG sample. The sample was divided into relaxed objects (cyan, offset $<0.2 R_{\text {vir }}$ and $v<0.5 v_{\text {disp }}$ ), high-velocity objects (yellow, offset $<0.2 R_{\text {vir }}$ and $v>0.5 v_{\text {disp }}$ ) and offset objects (green, offset $>0.2 R_{\mathrm{vir}}$ ). The black line represents the best-fit relation obtained through a Bayesian statistical analysis (the intrinsic scatter is labeled in the top-left corner). Right: $1.4 \mathrm{GHz}$ AGN radio power vs. temperature of the host group. The classification is the same as in the left panel. The grey line shows $1 \sigma$ errors on the best fit.

is strongly counterbalanced by the thermal pressure of the hot X-ray halo, which needs to be overcome to allow the inner SMBH feeding. As a result, hot-mode accretion is often feeble and $\sim 2$ orders of magnitude less intense compared with cold modes (e.g., Gaspari et al. 2013). Moreover, the hot accretion modes would develop a negative trend between the SMBH mass and plasma entropy/temperature $\left(\dot{M}_{\mathrm{B}} \propto K_{\mathrm{x}}^{-3 / 2} \propto T_{\mathrm{x}}^{-3 / 2}\right)$, with hotter halos accreting relatively less gas mass, which is ruled out by the observed SMBH mass versus X-ray halo scaling relations (Gaspari et al. 2019) and by our retrieved positive correlation $L_{\mathrm{R}}-T_{\mathrm{x}}$, a proxy for $P_{\mathrm{BH}}-T_{\mathrm{x}}$ (Sec. 3.4). Finally, no major duty cycle is expected from this mode. This is in conflict with a large intrinsic $\epsilon$ and with what is usually found in groups and clusters. There AGN feedback needs to rapidly suppress cooling of the hot halo via the AGN kinetic/radio power, i.e. establish frequent and efficient self-regulation (McNamara \& Nulsen, 2007; Gaspari et al., 2011; Fabian, 2012; Gitti et al., 2012; Prasad et al., 2015; Yang \& Reynolds, 2016).

Finally, we note that major mergers are unlikely to represent efficient triggers of the AGN and produce the scaling relations discussed here. The typical timescale of $\sim 5-6 \mathrm{Gyr}$ between two major mergers (e.g., Rodriguez-Gomez et al., 2015 ) is too long to provide steady support for the feeding of AGN. This is also consistent with Sharma et al. (2021), who recently showed that AGN activity is not enhanced by mergers. Moreover, we do not find substantive evidence for violent mergers in our sample. Nevertheless, mergers can still play a role over time in terms of supplying and preserving a significant amount of gas on the outskirts of the group halo.

\section{CONCLUSIONS}

We have carried out a comparison of the kinematic and optical properties of four different samples of COSMOS spectroscopic galaxy members: BGGs with and without radio emission, and satellites with and without radio emission. Scaling relations for the BGG samples were also investigated. Our results can be summarised as follows:

- Out of 70 BGGs, 56 ( $\sim 80 \%)$ are classified as ancient infallers, while the same fraction for satellites is only $\sim 42 \%$. We find that the fraction of ancient infaller among radio BGGs is $\sim 82 \%$, while the fraction of non-radio BGGs falling into this category is $\sim 78 \%$. This suggests that most BGGs, and in particular those hosting radio emission, have been accreted by the group at an early time.

- We find that radio galaxies with $L_{R}>10^{23} \mathrm{~W} \mathrm{~Hz}^{-1}$ always lie within $0.2 R_{\text {vir }}$ from the group centre, which has been defined as the X-ray emission peak. This is consistent with the current view of AGN feedback since the gas cooling out of the hot IGrM can feed the central SMBH, while outer galaxies need to rely on more episodic triggers.

- Our samples were compared to simulated BGGs from the HORIZON-AGN simulation. The ratio between the galaxy line-of-sight velocity and the group velocity dispersion for real BGGs (both radio and non-radio) shows a broader distribution than simulated galaxies, but still narrower than satellites. Statistical tests suggest that significant differences exist between simulated and real galaxies, indicating that additional physics may be needed to reproduce the true population of BGGs.

- We find that the stellar mass for radio BGGs is statisti- 
cally higher than for non-radio BGGs. This, in combination with the correlation between BGG mass and group mass, suggests that it is easier to find radio BGGs in higher-mass groups.

- We find positive correlations between the $1.4 \mathrm{GHz}$ power of radio $B G G s$ and the main properties of the diffuse $\mathrm{X}$-ray halo/intragroup medium, namely, $L_{\mathrm{R}}-T_{\mathrm{x}}$ and $L_{\mathrm{R}}-L_{\mathrm{x}}$, suggesting a link between AGN heating and cooling processes in the gaseous halo.

- We tested and discussed the two major AGN feeding/feedback scenarios. Our finding that galaxies at group centres are often radio galaxies better supports the CCA scenario since the AGN can feed from both the galactic and intragroup halo condensations via a flickering duty cycle. This is more difficult to explain in hot accretion modes (e.g., Bondi or ADAF), which can only tap into the nuclear $(r<100 \mathrm{pc})$ pressure-supported plasma region via continuous accretion. Unlike in hot modes, CCA becomes more vigorous in hotter and more luminous/massive halos. Thus, CCA naturally induces positive $L_{\mathrm{R}}-T_{\mathrm{x}}$ and $L_{\mathrm{R}}-L_{\mathrm{x}}$ correlations, as found in our samples.

\section{ACKNOWLEDGEMENTS}

We thank the referee for thoughtful comments that have improved the presentation of our results. AF thanks Gary Mamon and Stefano Borgani for insightful discussions. TP is supported by the BMBF Verbundforschung under grant number 50OR1906. MB acknowledges support from the Deutsche Forschungsgemeinschaft under Germany's Excellence Strategy - EXC 2121 "Quantum Universe" - 390833306. MG acknowledges partial support by NASA Chandra GO819104X/GO9-20114X and HST GO-15890.020-A grants.

\section{DATA AVAILABILITY}

The data underlying this article are available upon reasonable request.

\section{References}

Aubert D., Pichon C., Colombi S., 2004, MNRAS, 352, 376

Bahé Y. M., et al., 2017, MNRAS, 470, 4186

Balogh M. L., et al., 2011, MNRAS, 412, 2303

Barai P., Murante G., Borgani S., Gaspari M., Granato G. L., Monaco P., Ragone-Figueroa C., 2016, MNRAS, 461, 1548

Bassini L., et al., 2019, A\&A, 630, A144

Bassini L., et al., 2020, A\&A, 642, A37

Beers T. C., Flynn K., Gebhardt K., 1990, AJ, 100, 32

Bernardi M., 2007, AJ, 133, 1954

Bernstein J. P., Bhavsar S. P., 2001, MNRAS, 322, 625

Best P. N., Heckman T. M., 2012, MNRAS, 421, 1569

Best P. N., Kauffmann G., Heckman T. M., Ivezić Ž., 2005, MNRAS, 362, 9

Bondi H., 1952, MNRAS, 112, 195

Carlberg R. G., et al., 1997, ApJ, 485, L13

Cavaliere A., Santangelo P., Tarquini G., Vittorio N., 1986, ApJ, 305, 651
Coziol R., Andernach H., Caretta C. A., Alamo-Martínez K. A., Tago E., 2009, AJ, 137, 4795

Dubois Y., et al., 2014, MNRAS, 444, 1453

Eke V. R., Baugh C. M., Cole S., Frenk C. S., Navarro J. F., 2006, MNRAS, 370, 1147

Fabian A. C., 2012, ARA\&A, 50, 455

Fabian A. C., Canizares C. R., Boehringer H., 1994, ApJ, 425,40

Gaspari M., 2016, Proceedings of the International Astronomical Union, 11, 17-20

Gaspari M., Sa̧dowski A., 2017, ApJ, 837, 149

Gaspari M., Brighenti F., D'Ercole A., Melioli C., 2011, MNRAS, pp 1003-+

Gaspari M., Ruszkowski M., Oh S. P., 2013, MNRAS, 432, 3401

Gaspari M., et al., 2019, ApJ, 884, 169

Gaspari M., Tombesi F., Cappi M., 2020, Nature Astronomy, 4, 10

George M. R., et al., 2011, ApJ, 742, 125

George M. R., et al., 2012, ApJ, 757, 2

Giacintucci S., et al., 2011, ApJ, 732, 95

Giles P. A., et al., 2016, A\&A, 592, A3

Giodini S., et al., 2010, ApJ, 714, 218

Gitti M., Brighenti F., McNamara B. R., 2012, Advances in Astronomy, 2012

Gozaliasl G., et al., 2014, A\&A, 566, A140

Gozaliasl G., Finoguenov A., Khosroshahi H. G., Mirkazemi M., Erfanianfar G., Tanaka M., 2016, MNRAS, 458, 2762

Gozaliasl G., et al., 2019, MNRAS, 483, 3545

Gozaliasl G., et al., 2020, A\&A, 635, A36

Hamer S. L., et al., 2016, MNRAS, 460, 1758

Henden N. A., Puchwein E., Sijacki D., 2019, MNRAS, 489, 2439

Hernández-Fernández J. D., Haines C. P., Diaferio A., Iglesias-Páramo J., Mendes de Oliveira C., Vilchez J. M., 2014, MNRAS, 438, 2186

Hogan M. T., et al., 2015, MNRAS, 453, 1201

Ineson J., Croston J. H., Hardcastle M. J., Kraft R. P., Evans D. A., Jarvis M., 2013, ApJ, 770, 136

Ineson J., Croston J. H., Hardcastle M. J., Kraft R. P., Evans D. A., Jarvis M., 2015, MNRAS, 453, 2682

Jarvis M., et al., 2016, in MeerKAT Science: On the Pathway to the SKA. p. 6 (arXiv:1709.01901)

Jones L. R., Ponman T. J., Horton A., Babul A., Ebeling H., Burke D. J., 2003, MNRAS, 343, 627

Juráňová A., et al., 2019, MNRAS, 484, 2886

Kauffmann G., et al., 2003, MNRAS, 346, 1055

Kettula K., et al., 2013, ApJ, 778, 74

Kettula K., et al., 2015, MNRAS, 451, 1460

Kirkpatrick C. C., et al., 2021, MNRAS, 503, 5763

Kolokythas K., O'Sullivan E., Raychaudhury S., Giacintucci S., Gitti M., Babul A., 2018, MNRAS, 481, 1550

Laigle C., et al., 2019, MNRAS, 486, 5104

Lauer T. R., Postman M., Strauss M. A., Graves G. J., Chisari N. E., 2014, ApJ, 797, 82

Leauthaud A., et al., 2010, ApJ, 709, 97

Lilly S. J., et al., 2007, ApJS, 172, 70

Lin Y.-T., Mohr J. J., 2007, ApJS, 170, 71

Liu F. S., Xia X. Y., Mao S., Wu H., Deng Z. G., 2008, MNRAS, 385, 23

Liu F. S., Mao S., Deng Z. G., Xia X. Y., Wen Z. L., 2009, MNRAS, 396, 2003 
Liu W., et al., 2019, MNRAS, 484, 3376

Lovisari L., et al., 2020, ApJ, 892, 102

Mahajan S., Mamon G. A., Raychaudhury S., 2011, MN-

RAS, 416, 2882

Mahdavi A., Geller M. J., 2001, ApJ, 554, L129

Mamon G. A., 1992, ApJ, 401, L3

Mamon G. A., Biviano A., Boué G., 2013, MNRAS, 429, 3079

McDonald M., Gaspari M., McNamara B. R., Tremblay G. R., 2018, ApJ, 858, 45

McNamara B. R., Nulsen P. E. J., 2007, ARA\&A, 45, 117

Merloni A., Heinz S., 2007, MNRAS, 381, 589

Narayan R., Yi I., 1995, ApJ, 452, 710

Noble A. G., Webb T. M. A., Yee H. K. C., Muzzin A., Wilson G., van der Burg R. F. J., Balogh M. L., Shupe

D. L., 2016, ApJ, 816, 48

Padovani P., et al., 2017, A\&ARv, 25, 2

Pasini T., et al., 2020, MNRAS, 497, 2163

Peterson J. R., Fabian A. C., 2006, Phys. Rep., 427, 1

Ponman T. J., Allan D. J., Jones L. R., Merrifield M., McHardy I. M., Lehto H. J., Luppino G. A., 1994, Nature, 369, 462

Prasad D., Sharma P., Babul A., 2015, ApJ, 811, 108

Pulido F. A., et al., 2018, ApJ, 853, 177

Rhee J., Smith R., Choi H., Yi S. K., Jaffé Y., Candlish G.,

Sánchez-Jánssen R., 2017, ApJ, 843, 128

Rodriguez-Gomez V., et al., 2015, MNRAS, 449, 49

Rose T., et al., 2019, MNRAS, 489, 349

Sabater J., et al., 2019, A\&A, 622, A17

Saro A., Mohr J. J., Bazin G., Dolag K., 2013, ApJ, 772, 47

Sạdowski A., Gaspari M., 2017, MNRAS, 468, 1398

Schellenberger G., et al., 2020, ApJ, 894, 72

Schinnerer E., et al., 2010, ApJS, 188, 384

Shakura N. I., Sunyaev R. A., 1973, A\&A, 500, 33

Sharma R. S., et al., 2021, arXiv e-prints, p. arXiv:2101.01729

Shen S., Yang X., Mo H., van den Bosch F., More S., 2014, ApJ, 782, 23

Smolčić V., et al., 2007, ApJS, 172, 295

Stott J. P., Edge A. C., Smith G. P., Swinbank A. M., Ebel-

ing H., 2008, MNRAS, 384, 1502

Stott J. P., et al., 2010, ApJ, 718, 23

Temi P., Amblard A., Gitti M., Brighenti F., Gaspari M., Mathews W. G., David L., 2018, ApJ, 858, 17

Teyssier R., 2002, A\&A, 385, 337

Tremblay G. R., et al., 2018, ApJ, 865, 13

Truong N., Pillepich A., Werner N., 2021, MNRAS, 501, 2210

Voit G. M., Ma C. P., Greene J., Goulding A., Pandya V., Donahue M., Sun M., 2018, ApJ, 853, 78

Von Der Linden A., Best P. N., Kauffmann G., White S. D. M., 2007, MNRAS, 379, 867

Wittor D., Gaspari M., 2020, MNRAS, 498, 4983

Wu X.-P., Xue Y.-J., Fang L.-Z., 1999, ApJ, 524, 22

Yang H. Y. K., Reynolds C. S., 2016, ApJ, 818, 181

Yang H. Y. K., Gaspari M., Marlow C., 2019, ApJ, 871, 6

Zhang Y. Y., Andernach H., Caretta C. A., Reiprich T. H., Böhringer H., Puchwein E., Sijacki D., Girardi M., 2011, A\&A, 526, A105 